seldorf 2012 zu einer Beitragserhöhung und damit zu einer außerordentlichen Steigerung der Abgeordnetenbezüge. Deren Erhöhung wäre grundsätzlich eine Voraussetzung für einen entsprechenden Systemwechsel der Altersversorgung. Zuletzt hat die Unabhängige Kommission des Bundestages es einstimmig abgelehnt - aus Kosten-, Unsicherheits- sowie Gleichheitsgründen und nicht zuletzt auch aus der Überlegung, die Altersversorgung für gemeinwohldienliche öffentliche Ämter nicht ins Privatwirtschaftliche samt den damit verbundenen Risiken für die Amtsträger abzuschieben. ${ }^{37}$

Auch diese Überlegung stützt sich auf die eine Grundtatsache, die für alle Überlegungen zum finanziellen Status des Abgeordneten maßgeblich ist: Er ist Inhaber eines öffentlichen Amtes sui generis. Dem sollte er sich allerdings auch würdig erweisen: durch Kompetenz, Seriosität, Stil und nicht zuletzt auch durch den Mut, angemessene Amtsausstattung mit den Bürgern zu kommunizieren. Nicht nur in Bayern besteht auf all diesen Feldern Verbesserungsbedarf.

37 Vgl. BT-Drs. 17/12500, S. 22 f. Sehr detaillierte Empfehlungen und Materialien zugunsten eines Versorgungswerks: Sächsischer Landtag, Bericht der Sachverständigenkommission zur Erarbeitung von Vorschlägen für die Neuregelung des Gesetzes über die Rechtsverhältnisse der Mitglieder des Sächsischen Landtages, Dresden 2006.

\title{
Die bayerische Landtagswahl vom 15. September 2013: Bund und Land Hand in Hand
}

\author{
Rainer-Olaf Schultze
}

Im Frühherbst 2013 standen in Bayern innerhalb einer Woche gleich mehrere Wahlentscheidungen an: Am 15. September wählten die Bayern die 180 Abgeordneten ihres Landtages; am 22. September fand die Bundestagswahl statt. ${ }^{1}$ Am Tag der Landtagswahl bestellten die bayerischen Wähler zudem die Mitglieder der Bezirksräte und stimmten über fünf Änderungsvorschläge zur bayerischen Verfassung ab. In der Termingestaltung setzte die CSU ihr wahlstrategisches Interesse gegenüber ihrem liberalen Koalitionspartner in der Staatsregierung wie gegenüber der Opposition in Bund wie Land durch: Es ging der CSU erstens um zwei Wahltermine an aufeinander folgenden Sonntagen und bald nach dem Ende der Sommerferien Anfang September (und damit um einen kurzen Wahlkampf), so

1 Die wahlstatistische Dokumentation des Beitrags beruht auf der offiziellen Veröffentlichung des Bayerischen Landesamtes für Statistik und Datenverarbeitung, Wahl zum Bayerischen Landtag am 15. September 2013. Endgültiges Ergebnis, München 2013. Außerdem wurden Daten von den Webseiten des Landesamtes entnommen (www.statistik.bayern.de). Der wahlsoziologischen Interpretation liegen zugrunde: Infratest dimap, Wahlreport. Landtagswahl in Bayern am 15. September 2013, Berlin 2013; Forschungsgruppe Wahlen, Wahl in Bayern. Eine Analyse der Landtagswahl vom 15. September 2013, Berichte Nr. 153, Mannheim 2013; Auswertungen der SZ und der Augsburger Allgemeinen (AZ). 
dass die Wahlen zu Bund und Land nicht am selben Tag stattfinden würden (also anders als in Hessen); und zweitens sollte zunächst die Wahl zum Bayerischen Landtag abgehalten werden - in der Diktion Horst Seehofers „die Mutter aller Schlachten“.

Diese Wahlstrategie ging auf: Die CSU wetzte bei der Landtagswahl mit einem Stimmenanteil von 47,7 Prozent zu einem Gutteil die Scharte ihrer drastischen Verluste von 2008 aus und stellt neuerlich mit absoluter Mandatsmehrheit im Maximilianeum die Alleinregierung im Freistaat. ${ }^{2}$ Zudem gewann sie nach deutlichen Einbußen an absoluten Stimmen - über mehrere Wahlen aller vier Ebenen hinweg - erstmalig auf Landes- wie Bundesebene in beachtlichen Zahlen Wähler hinzu und erreichte bei der Bundestagswahl mit 49,3 Prozent der Zweitstimmen fast die magische Zahl von 50 plus x.

Wahlkampf und Wählerentscheidung beider Wahlen stehen - in Anbetracht der Verzahnung bundesrepublikanischer Politikverflechtung nicht sonderlich überraschend - offenkundig in einem engen Zusammenhang. In ihm manifestiert sich zudem das spezifisch bayerische Bund-Länder-Verhältnis, untermauert durch die institutionelle Doppelrolle der CSU als bayerischer „Staats- und Ordnungspartei“ (Alf Mintzel) und als eigenständige, im Fraktionsbündnis mit der CDU agierende Bundespartei. Es gilt also nicht nur die Landtagswahl in den Blick zu nehmen und entlang der wahlsoziologischen Determinanten-Trias die relative Bedeutung von Langfrist- und Kurzzeitfaktoren für die bayerische Wählerentscheidung zu bestimmen; vielmehr müssen die Wahlen der verschiedenen Arenen betrachtet und nach den wechselseitigen Einflüssen gefragt werden. Dabei geht es weniger um die wohlfeilen, parteipolitischen oder auch persönlichen Opportunitäten folgenden Interpretationen am Wahlabend - bei Niederlagen oder Stimmenverlusten meist in Form exkulpierender Schuldzuweisungen an die Adresse der Akteure der jeweils anderen politischen Arena; es geht vielmehr um die Antwortversuche der Wahlforschung, die sich nach langer Zeit geringen Interesses wieder vermehrt der Analyse des Einflusses der Wahlebenen und insbesondere der Bundes- und Landtagswahlen in ihren Wechselwirkungen auf die jeweiligen Wählerentscheidungen zugewandt hat (siehe unten).

\section{Ausgangslage}

Mit ihrem Regierungsprogramm „Zukunft gemeinsam gewinnen“ vom Dezember 2008 unternahm die CSU/FDP-Koalitionsregierung unter Ministerpräsident Horst Seehofer und dem FDP-Wirtschaftsminister Martin Zeil den Versuch eines Neuanfangs, im Politikstil wie in den Inhalten: So setzte man koalitionsintern (zunächst) auf Gemeinsamkeit, versprach, auf die Bürger zuzugehen, und die CSU versuchte, sich als „Mitmach-Partei“ zu öffnen; sachpolitisch ging man daran, relativ geräuschlos eine Reihe von Altlasten abzuräumen. ${ }^{3}$ Dazu gehörte nicht zuletzt die Aufarbeitung des Fiaskos um die Bayern LB, indem

2 Zur vorangegangenen Wahl von 2008 siehe Rainer-Olaf Schultze / Jan Grasnick, Die bayerische Landtagswahl vom 28. September 2008: Betriebsunfall oder Ende eines Mythos?, in: ZParl, 40. Jg. (2009), H. 1, S. 34 - 55; vgl. auch den Überblick von Harald Schoen, Landtagswahlen in Bayern 1966 bis 2003: Verstärkte bundespolitische Durchdringung aufgrund der Doppelrolle der CSU, in: ZParl, 38. Jg. (2007), H. 3, S. $503-512$.

3 Zur Entwicklung der CSU in der jüngeren Vergangenheit siehe Gerhard Hopp / Martin Sebaldt I Benjamin Zeitler (Hrsg.), Die CSU. Strukturwandel, Modernisierung und Herausforderungen einer Volkspartei, Wiesbaden 2010; Michael Weigl, Die CSU. Akteure, Entscheidungsprozesse 
man durch Verhandlungen mit der österreichischen Regierung die marode Kärntner Bank Hypo-Alpe-Adria los wurde und die Belastungen für den Staatshaushalt in Grenzen hielt. ${ }^{4}$ Hinzu kamen flexiblere Lösungen auf dem Felde der Infrastrukturpolitik, indem man den Bürgern etwa in der Frage des Donauausbaus oder weiterer Großbauprojekte entgegenkam. Überhaupt waren Seehofer und die Staatsregierung während der gesamten Wahlperiode bemüht, die Bürgerbegehren - anders als in der Vergangenheit - ernst zu nehmen: Entweder wurden sie umgesetzt, wie der Volksentscheid für ein strengeres Rauchverbot vom Juli $2010^{5}$, oder sie wurden - wenn auch in einigen Fällen widerstrebend - akzeptiert, zum Beispiel beim Bau der dritten Startbahn des Münchener Flughafens, die am Einspruch der Münchener Bürger ebenso scheiterte ${ }^{6}$ wie die von der Stadt- wie Landesregierung unterstützten Bewerbungsabsichten für die Durchführung olympischer Winterspiele in München und Oberbayern.

Die Konsolidierung von Regierung und Partei war spätestens seit dem Sommer 2011 erfolgreich abgeschlossen. ${ }^{7}$ Bundespolitisch trug dazu nicht zuletzt das Ende der Plagiatsaffäre um Karl Theodor Baron zu Guttenberg durch dessen Rücktritt als Bundesverteidigungsminister und sein Ausscheiden aus der aktiven Politik bei; Guttenberg galt zeitweilig als Hoffnungsträger der Partei und durchaus auch als möglicher Herausforderer Seehofers ${ }^{8}$; sodann die Wende in der Energiepolitik mit den Beschlüssen zum Atomausstieg im Gefolge der Reaktorkatastrophe in Fukushima. Landespolitisch profitierte man von der guten Wirtschaftslage Bayerns mit ihren überdurchschnittlichen Wachstumsraten und den in weiten Landesteilen, vor allem im oberbayerischen Speckgürtel um München, überdurchschnittlichen Einkommensverhältnissen bei gleichzeitig deutlich geringerer Arbeitslosigkeit. Dies vergrößerte die Handlungsspielräume der Politik und erlaubte der Staatsregierung in den Doppelhaushalten ab 2011/2012 sowohl eine expansive Haushaltspolitik als auch den Beginn der Schuldentilgung in Höhe von jährlich einer Milliarde Euro. ${ }^{9}$ Die demoskopischen

und Inhalte einer Partei am Scheideweg, Baden-Baden 2013, sowie die kenntnisreiche Analyse Gerhard Hirscher, Wohin geht die bürgerliche Mehrheit? Die Unionsparteien in den Wahlen 2010 bis 2013, Hanns Seidel Stiftung, Berichte \& Studien 98, München 2014.

4 Zum Verkauf an die Republik Österreich vgl. „Das Landesbank-Desaster in Zahlen“, in: AZ vom 15. Dezember 2009, S. 1; Mariele Schulze, Österreich fängt die Pleitebank auf, in: AZ vom 15. Dezember 2009, S. 4, Josef Karg, Milliarden-Pleite: Landesbank-Chef muss gehen, in: AZ vom 15. Dezember 2009, S. 1; Bayern LB, „BayernLB gibt Anteile an der Hypo Group Alpe Adria (HGGA) an die Republik Österreich ab", Pressemitteilung vom 14. September 2009, http:// www.bayernlb.de/internet/de/content/metanav/investor_relations/veroeffentlich_1/ir_releases_4/ ir_news/news_18954.jsp (Abruf am 9. Mai 2014).

5 Vgl. Karel Auer / Mike Szymanski / Peter Fahrenholz, Triumph der Nichtraucher, in: SZ vom 5. Juli 2010, S. 1; Gerhard Hirscher, a.a.O. (Fn. 3), S. 177.

6 Vgl. Nina Bovensiepen / Frank Müller / Silke Lode, Münchner gegen dritte Startbahn, in: Süddeutsche.de vom 18. Juni 2012, http://www.sueddeutsche.de/muenchen/muenchner-stoppen-drittestartbahn-landesregierung-will-an-flughafenausbau-festhalten-1.1385544 (Abruf am 9. Mai 2014).

7 Vgl. Gerhard Hirscher, a.a.O. (Fn. 3), S. $173 \mathrm{ff}$.

8 Vgl. die Analyse der SZ-Journalisten Roland Preuß/ Tanjev Schultz, Guttenbergs Fall. Der Skandal und seine Folgen für Politik und Gesellschaft, Gütersloh 2011.

$9 \mathrm{Zu}$ den Etats siehe die Veröffentlichungen des Finanzministeriums unter http://www.stmf.bayern. de/haushalt/ (Abruf am 9. Mai 2014); vgl. auch Bayerische Staatsregierung, „Bayern 2030 schuldenfrei“, http://www.bayern.de/Bayern-2030-schuldenfrei-.4622/index.htm (Abruf am 9. Mai 2014). 
Daten spiegeln dies wider. Neun von zehn bayerischen Wählern sehen den Freistaat wirtschaftlich an der Spitze der Bundesländer; zwei Drittel schätzten ihre persönliche wirtschaftliche Lage im Vorfeld der Wahlen von 2013 als gut ein (2008: 48 Prozent), während nur noch 28 Prozent ihre Situation als teils gut, teils schlecht bezeichneten (2008: 40 Prozent). Gleichfalls zwei Drittel der Befragten brachten die Wirtschaftsentwicklung dabei mit der CSU in Verbindung. ${ }^{10}$

\section{Wahlkampf und Meinungsklima}

Ab dem Jahreswechsel 2011/2012 prognostizierten die Demoskopen der CSU jedenfalls durchweg Stimmenanteile von 46 Prozent und darüber ${ }^{11}$; die Zufriedenheit mit der Arbeit der Staatsregierung, die im September 2008 bei 51 Prozent gelegen hatte, stieg bis zur Landtagswahl kontinuierlich auf 65 Prozent an. ${ }^{12}$ Die Wähler rechneten ihre Zustimmung dabei überwiegend der CSU zu. Während zwei Drittel von ihnen mit der Arbeit der Partei in der Regierung zufrieden waren, zeigten sich 78 Prozent mit der Leistung des Koalitionspartners FDP weniger oder gar nicht zufrieden. Gleichfalls drei Viertel der Befragten stimmten der Aussage zu, von den Liberalen habe man in der Staatsregierung nicht viel gemerkt; 58 Prozent waren der Meinung, dass diese in den letzten Jahren nichts bewegt hätten. Jedenfalls verfestigte sich im Laufe der Wahlperiode dieser Eindruck in der öffentlichen Wahrnehmung. Er fand seine besondere Bestätigung im Frühjahr 2013 in dem Koalitionsstreit um die Studiengebühren; während die FDP-Minister Martin Zeil und Wolfgang Heubisch im Einklang mit dem Koalitionsvertrag an ihnen festhalten wollten, vollzogen Seehofer und die CSU unter dem Eindruck des erfolgreichen Volksbegehrens - initiiert von den Freien Wählern und getragen auch von den übrigen Oppositionsparteien - eine Kehrtwende und setzten deren Abschaffung durch, um sich nicht der Gefahr einer Niederlage bei der ansonsten anstehenden Volksabstimmung auszusetzen. ${ }^{13}$ Spätestens von diesem Zeitpunkt an setzte die CSU-Führung - zumindest unausgesprochen - nicht mehr auf die Fortführung der Koalition mit der FDP, sondern auf die Alleinregierung, dies umso mehr, als die liberale Landespartei auch vom desolaten Erscheinungsbild der Bundes-FDP negativ betroffen war. Nur noch 42 Prozent der Befragten befürworteten in der Infratest dimapWahltagbefragung den Einzug der Liberalen in den Landtag; 2008 waren es immerhin zwei Drittel gewesen. ${ }^{14}$

10 Laut Wahltagbefragung der Forschungsgruppe Wahlen stimmten 69 Prozent der Aussage zu, „dass Bayern wirtschaftlich gut dasteht, liegt vor allem an der Politik der CSU“; vgl. Forschungsgruppe Wahlen, a.a.O. (Fn. 1), S. 25 ff.

11 Vgl. Gerhard Hirscher, a.a.O. (Fn. 3), S. 135 ff., S. $173 \mathrm{ff}$.

12 Diese und die folgenden Daten nach Infrastest dimap, a.a.O. (Fn. 1), S. 17 ff.

13 Zum Ergebnis des Volksbegehrens zur Abschaffung der Studiengebühren vgl. Martina Scherf, Triumph in letzter Minute, in: SZ von 31. Januar 2013, S. 36; Johann Osel, Die 1000-Euro-Frage, in: SZ vom 1. Februar 2013, S. 4. Zum Koalitionsstreit, zur Kehrtwende der CSU und dem Einknicken der FDP aus Koalitionsräson vgl. exemplarisch aus der Berichterstattung im Februar und März 2013 Frank Müller / Mike Szymanski, Kompromiss soll Koalitionsstreit beenden, in: SZ vom 8. Februar 2013, S. 45; „Studiengebühren stürzen FDP in die Krise“, in: SZ vom 15. Februar 2013, S. 45; Frank Müller, Ein Friede für 900 Millionen Euro, in: SZ vom 25. Februar 2013, S. 24; Mike Szymanski, Stich in die liberale Seele, in: SZ vom 4. März 2013, S. 32.

14 Vgl. Infrastest dimap, a.a.O. (Fn. 1), S. 17 ff. 
Die drei Oppositionsparteien, Sozialdemokraten, Bündnis 90/Die Grünen und Freie Wähler, glaubten dennoch zu verschiedenen Zeitpunkten während der Wahlperiode an die Chance, die amtierende CSU/FDP-Koalition gemeinsam ablösen zu können. Insbesondere im Jahr 2011 schienen sie sich unter dem Eindruck der Reaktorkatastrophe und der Ankündigung des Münchener Oberbürgermeisters Christian Ude, als Spitzenkandidat der SPD Seehofer als Ministerpräsidenten herauszufordern, deutlich im Aufwind zu befinden. Die Demoskopen signalisierten den drei Parteien steigende Umfragewerte ${ }^{15}$; sie taxierten SPD wie Grüne zeitweilig bei 20, die Freien Wähler bei zehn bis zwölf Prozent, so dass eine Machtchance im Bereich des Möglichen zu sein schien. Tatsächlich kamen die Spitzen der drei Parteien verschiedentlich zu Sondierungsgesprächen zusammen, die allerdings eher die tiefgreifenden Differenzen zutage förderten, denn sozial wie kulturell passen die drei Parteien in Wähler- wie Mitgliederbasis kaum zusammen, und sie unterscheiden sich deshalb programmatisch auch deutlich voneinander. Zudem sanken in dem Maße, in dem die CSU sich konsolidierte, die Umfragewerte und Aussichten der Opposition deutlich, so dass es sich nur um wenig erfolgversprechende Momentaufnahmen handelte. Vor allem aber bestehen unverändert die kulturell wie regional tief verwurzelten Antipathien in großen Segmenten der Wählerschaft - Barrieren, die weder Sozialdemokraten noch Grüne zu überwinden vermögen, wenn fast zwei Drittel der Wähler der Auffassung sind, die SPD passe nicht zu Bayern, und nur ein Viertel der Wähler den Sozialdemokraten zutraut, die Probleme besser lösen zu können als die amtierende Regierung. ${ }^{16}$

Auch die Kandidatur Udes vermochte daran nichts Entscheidendes zu ändern - im Gegenteil: Zwar verfügten Ude und Seehofer mit 65 Prozent über ein gleich hohes Maß an Wählerzufriedenheit und lagen damit deutlich vor den anderen Spitzenkandidaten Hubert Aiwanger (39), Magerete Bause (30) und Martin Zeil (25 Prozent). Galt Ude als sympathischer, glaubwürdiger und kompetenter in Fragen sozialer Gerechtigkeit, so bescheinigten die Wähler Seehofer die größere Durchsetzungsfähigkeit im Bund wie im Land; vor allem aber sei er derjenige von beiden, der eher zu Bayern passe, während Ude als Münchener Oberbürgermeister insbesondere außerhalb Münchens und auf dem flachen Lande auf Skepsis oder auch Ablehnung stieß (siehe Tabelle 1). Die Trias von Parteibindung, Kandidatenpräferenz und Sachkompetenzen als Erklärungsvariablen der Wählerentscheidung zugrunde legend, gelangte die Forschungsgruppe Wahlen zu folgendem asymmetrischen Ergebnis: Für die SPD-Wahlentscheidung sei „die Parteibindung der mit Abstand stärkste Einflussfaktor, gefolgt von der parteispezifischen Kompetenzzuschreibung, abgeschlagen ist die Kandidatenpräferenz “, während im Falle der CSU-Wähler die Ministerpräsidentenpräferenz und die Parteibindung als Entscheidungsfaktor in etwa gleichauf lägen. ${ }^{17}$

Die ungleiche Kandidatenalternative, die große Zufriedenheit mit der Staatsregierung und der CSU, das hohe Maß an Zukunftsvertrauen - immerhin sind 85 Prozent laut Wahltagbefragung der Forschungsgruppe Wahlen davon überzeugt, dass Bayern gut auf die $\mathrm{Zu}$ kunft vorbereitet sei - manifestierten sich auch im teils großen Kompetenzvorsprung der CSU auf den meisten Politikfeldern, auch den landespolitischen. Dies galt für CSU-Mehrheiten bei der Wirtschafts-, Infrastruktur- und Verkehrspolitik, aber auch für die Arbeitsmarktpolitik, wo die Wähler gleichfalls in ihrer Mehrheit es eher der CSU zutrauten, neue

15 Vgl. Gerhard Hirscher, a.a.O. (Fn. 3), passim.

16 Vgl. Forschungsgruppe Wahlen, a.a.O. (Fn. 1), S. 14; Infratest dimap, a.a.O. (Fn. 1), S. 36.

17 Vgl. Forschungsgruppe Wahlen, a.a.O. (Fn. 1), S. 28. 


\begin{tabular}{|l|c|c|}
\hline Tabelle 1: Profile der Spitzenkandidaten vor der Landtagswahl 2013 (in Prozent) \\
\hline & Horst Seehofer & Christian Ude \\
\hline ist die stärkere Führungspersönlichkeit & 74 & 14 \\
kann die Interessen Bayerns im Bund besser vertreten & 72 & 17 \\
passt eher zu Bayern & 69 & 22 \\
versteht mehr von der Wirtschaft & 61 & 16 \\
gibt in der Öffentlichkeit eine bessere Figur ab & 55 & 34 \\
setzt sich eher für die Schaffung neuer Arbeitsplätze ein & 52 & 26 \\
ist sympathischer & 41 & 46 \\
ist besser mit den Problemen der Bürger vertraut & 39 & 41 \\
ist glaubwürdiger & 34 & 43 \\
setzt sich stärker für soziale Gerechtigkeit ein & 28 & 52 \\
\hline Frage: Wenn Sie einmal Horst Seehofer und Christian Ude miteinander vergleichen: Wer von beiden ...? \\
Grundgesamtheit: Wahlberechtigte Bevölkerung in Bayern; fehlende Werte zu 100 Prozent: Spontan: \\
keiner von beiden / weiß nicht / keine Angabe. \\
Quelle: Eigene Zusammenstellung nach Infratest dimap, a.a.O. (Fn. 1), S. 34. \\
\hline
\end{tabular}

Arbeitsplätze zu schaffen. Vorne lag die Union selbstredend auf dem Feld der Familienpolitik, aber auch, eher überraschend und jeweils mit nur geringem Vorsprung vor der SPD, in der Schul- und Bildungspolitik (36 zu 24 Prozent) und in der Frage der sozialen Gerechtigkeit (von 33 zu 28 Prozent) wie auf dem Feld der Energiepolitik knapp vor Bündnis 90/ Die Grünen (32 zu 30 Prozent). ${ }^{18}$ Etwas andere Ergebnisse ermittelte allerdings Infratest dimap in ihrer Wahltagbefragung. Dort rangierten die Sozialdemokraten vor der Union auf dem Feld der Lohnpolitik (44 zu 28 Prozent) wie in der Frage sozialer Gerechtigkeit (42 zu 29 Prozent) und die Grünen bei der Umwelt- und Klimapolitik deutlich vor der Union (52 zu 22 Prozent). ${ }^{19}$

Die Ergebnisse der Wahltagbefragungen zeigen aber auch, wie wenig die Wähler zwischen bundes- und landespolitischen Themen unterscheiden, ja aufgrund der Politikverflechtung und der beiden benachbarten Wahltermine überhaupt unterscheiden können. Die vom Wähler ausweislich der Daten in Tabelle 3 als sehr wichtig wahrgenommenen Themen fallen mit Ausnahme der Wohlstandssicherung und der Verwandtenaffäre alle in die Bundeskompetenz; ähnliches gilt mit Ausnahme der Bildungs- und regionalen Verkehrspolitik für die in Tabelle 2 aufgelisteten Daten zur Einschätzung des politischen Problemhaushaltes. Zudem trafen viele der Positionsbestimmungen von Sozialdemokraten und Bündnis 90/Die Grünen im Bundestagswahlkampf, etwa in Sachen Steuerpolitik, auf Misstrauen und Ablehnung bei den bayerischen Wählern.

Den Oppositionsparteien erschwerte dies die Wahlkampfführung, da sie ihre Strategie auf die Auseinandersetzung um landespolitische Themen und insbesondere um die Versäumnisse wie die tatsächlichen und vermeintlichen Skandale von Regierung und CSU ausgerichtet hatten. Letztere spielten im Wahlkampf wie in der Wählerentscheidung nur eine überraschend geringe Rolle. Dies gilt für die Justizaffäre um Gustl Mollath ${ }^{20}$ und für ver-

18 Vgl. ebenda, S. $21 \mathrm{ff}$.

19 Vgl. Infratest dimap, a.a.O. (Fn. 1), S. 17 ff.

20 Siehe den Schlussbericht des Untersuchungsausschusses des Bayerischen Landtages vom 10. Juli 2013, Bayerischer Landtag, Drs. 16/17741; Uwe Ritzer / Olaf Przybilla, Die Affäre Mollath. Der Mann, der zu viel wusste, München 2013. 


\begin{tabular}{|l|c|c|c|}
\hline \multicolumn{4}{|c|}{ Tabelle 2: Die wichtigsten politischen Probleme in Bayern vor der Landtagswabl (in Prozent) } \\
\hline & 2013 & 2008 & 2003 \\
\hline Bildung / Schule / Ausbildung & 31 & 46 & 18 \\
Familienpolitik / Kinderbetreuung & 16 & 11 & 6 \\
Arbeitslosigkeit / Arbeitsmarkt & 15 & 28 & 65 \\
Wirtschaftliche Situation & 10 & 13 & 24 \\
Rentenniveau / Alterssicherung & 8 & & \\
Soziale Ungerechtigkeit & 8 & & \\
Verkehr / S-Bahn / Flughafenausbau & 8 & \\
Mindestlohn / Lohnpolitik & 7 & \\
PKW-Maut & 7 & & \\
\hline Grundgesamtheit: Wahlberechtigte Bevölkerung in Bayern; Mehrfachnennungen möglich. \\
Quelle: Infratest dimap, LTW 2008, a.a.O. (Fn. 37), S. 25 f.; dies., LTW 2013, a.a.O. (Fn. 1), S. 22. \\
\hline
\end{tabular}

\begin{tabular}{|l|c|}
\hline Tabelle 3: Themen und Wablentscheidung vor der Landtagswabl 2013 (in Prozent) \\
\hline & sehr wichtig \\
\hline angemessene Löhne und Arbeitsbedingungen & 58 \\
eine gute Absicherung im Alter & 56 \\
die künftige Energieversorgung & 50 \\
die Zukunft des Euro & 35 \\
die Sicherung des Wohlstands in Bayern & 32 \\
der Streit um die PKW-Maut für ausländische Autofahrer & 17 \\
der politische Streit um die Überwachung durch Geheimdienste & 17 \\
die Affäre um die Beschäftigung von Verwandten durch Landtags- \\
abgeordnete und Minister \\
\hline Frage: Ich nenne Ihnen jetzt einige politische Themen. Bitte sagen Sie mir jeweils, wie wichtig das jeweili- \\
ge Thema für Ihre Wahlentscheidung ist. Würden Sie sagen ... ist / sind für Ihre Wahlentscheidung ...? \\
Grundgesamtheit: Wahlberechtigte Bevölkerung in Bayern; fehlende Werte zu 100 Prozent: weiß nicht / \\
keine Angabe / wichtig / weniger wichtig / gar nicht wichtig. \\
Quelle: Eigene Zusammenstellung nach Infratest dimap, a.a.O. (Fn. 1), S. 36. \\
\hline
\end{tabular}

schiedene weitere, mindestens grenzwertige Verfilzungen regionaler wie lokaler Mandatsund Amtsträger, vor allem aber für die Diäten- und Verwandtenaffäre um die (seit 2001 nicht mehr zulässige) Beschäftigung von Familienangehörigen durch Landtagsabgeordnete und deren Bezahlung durch den Steuerzahler, die im Frühjahr 2013 offenbar wurde. ${ }^{21}$ Betroffen davon waren überwiegend Mandatsträger der CSU, einige aus der Ministerriege und eine Reihe führender Fraktionsmitglieder, an ihrer Spitze der Fraktionsvorsitzende Georg Schmid. Um möglichen Schaden bei der Landtagswahl im Herbst abzuwenden, reagierte die Parteiführung umgehend; Seehofer veranlasste die betreffenden Kabinettsmitglieder zur Zu-

21 Zur Diäten- und Verwandtenaffäre siehe den Beitrag von Heinrich Oberreuter in diesem Heft der ZParl. Zum Verlauf im Einzelnen vgl. die (fast) tägliche Berichterstattung im Bayernteil der SZ ab Mitte April 2013; unter anderem Frank Müller / Mike Szymanski, Die Familienhilfe der CSU, in: SZ vom 19. April 2013, S. 49; dies., „Der Chef lässt kündigen“, in: SZ vom 20. April 2013, S. 48. 
rückzahlung der Gelder und Schmid zum Rücktritt vom Fraktionsvorsitz; als Platzhalterin amtierte bis zur Neukonstituierung des Landtags nach der Wahl Christa Stewens. ${ }^{22}$

Zwar lasteten die bayerischen Wähler Skandale, Affären und Filz der CSU durchaus an; Wechselstimmung löste dies während des Wahlkampfes allerdings nicht aus. Die führende Rolle der Union geriet nicht in Gefahr. Offen war am Vorabend der Wahl zweierlei: erstens, ob es für die CSU zur absoluten Mandatsmehrheit reichen würde, zumal eine CSU-Alleinregierung von der Mehrheit der Wähler mit 58 zu 37 Prozent abgelehnt wurde und gleichfalls eine Mehrheit von 52 zu 42 Prozent der Überzeugung war, dass es dazu nicht kommen würde ${ }^{23}$; zweitens, wer gegebenenfalls als Koalitionspartner zur Verfügung stünde, die Liberalen, sofern sie nicht an der Sperrklausel scheiterten, oder die Freien Wähler, die unter Führung von Hubert Aiwanger und Michael Piazolo, obwohl sie sich in ihren öffentlichen Einlassungen während der Wahlkampfes bedeckt hielten, wohl doch zum Einstieg in die Regierung bereitstehen würden.

\section{Wablergebnis}

Zur Landtagswahl 2013 waren über 9,4 Millionen Bayern wahlberechtigt. Im Vergleich zu den Wahlen von 2003 und 2008 stieg die Wahlbeteiligung deutlich auf 63,6 Prozent (+5,7 Prozentpunkte), blieb allerdings um mehr als sechs Punkte hinter der Wahl von 1998 zurück und wies regionale wie lokale Unterschiede auf, von denen beachtliche Wirkungen auf die Stimmstärken der Parteien ausgingen (siehe unten). Bei der Bundestagswahl erreichte die Beteiligung in Bayern 70,0 Prozent und blieb damit um 1,6 Prozentpunkte hinter der von 2009 zurück. Von der Möglichkeit der Briefwahl machten mit 36,8 Prozent mehr als ein Drittel der Wähler Gebrauch, die damit vom Bürger nicht mehr nur als Ausnahme, sondern als Regelverfahren begriffen und auch praktiziert wird. Dadurch konzentriert sich der Zeitraum der Stimmabgabe nicht mehr auf einen Tag, sondern erstreckt sich über mehrere Wochen - durchaus nicht unproblematisch, sofern sich in Anbetracht unserer schnelllebigen Zeit die Entscheidungssituation der Wähler vor dem Wahltag kurzfristig tiefgreifender verändern sollte. ${ }^{24}$ Bei Landtags- wie Bundestagswahl traten diesmal allerdings nur vergleichsweise geringe Differenzen in den Stimmenanteilen der Parteien bei Urnen- und Briefwählern auf: Sie differierten bei der Landtagswahl für die Parteien durchweg unter einem Prozentpunkt, eine Woche später bei der Bundestagswahl bei über einem und bis zu zwei Prozentpunkten, wobei SPD und Freie Wähler bei den Briefwählern in beiden Wahlen schlechter, Grüne und FDP jeweils besser abschnitten, während die CSU einen Prozentpunkt hinter dem Stimmenanteil bei den Urnenwähler zurückblieb.

Das Ergebnis selbst war, anders als 2008, eindeutig. Gewinner waren in beiden Fällen Horst Seehofer und die CSU, während die „kleinen“ Parteien, die 2008 fast 40 Prozent der

22 Siehe die Berichte im Bayernteil der SZ; unter anderem Stefan Mayr / Frank Müller / Mike Szymanski, Stewens - ein Comeback auf Zeit, in: SZ vom 26. April 2013, S. 46; Frank Müller / Mike Szymanski, Schmid geht, der Ärger bleibt, in: SZ vom 27. April 2013, S. 47.

23 Vgl. Forschungsgruppe Wahlen, a.a.O. (Fn. 1), S. 15.

24 Zur nächsten Landtagswahl 2018 bereitet die Staatsregierung - laut Finanzminister Markus Söder - die Einführung des „electronic voting“ vor; vgl. „Söder will Bürger im Internet abstimmen lassen“, in: Süddeutsche.de vom 12. Januar 2014, http://www.sueddeutsche.de/bayern/wahlen-inbayern-soeder-will-buerger-im-internet-abstimmen-lassen-1.1860885 (Abruf am 9. Mai 2014). 
Landtagswahl-Stimmen erhalten hatten, sämtlich schlechter abschnitten. Dies gilt vor allem für die Liberalen, die nach ihrem fünfährigen Zwischenspiel in Parlament und Regierung mit 3,3 Prozent wieder einmal an der Sperrklausel scheiterten, aber auch für Freie Wähler und Grüne, die gegenüber 2008 jeweils 1,2 Prozentpunkte und mit 18 beziehungsweise 19 Mandaten zwei beziehungsweise einen Sitz im Landtag einbüßten und damit deutlich hinter ihren eigenen Erwartungen zurückblieben. Ähnlich erging es den außerparlamentarischen Oppositionsparteien, von denen die Linkspartei rund die Hälfte ihrer Wählerschaft verlor und auf einen Stimmenanteil von 2,1 Prozent zurückfiel. Insgesamt wurden 14,0 Prozent der Gesamtstimmen für Parteien abgegeben, die unter der Fünf-Prozent-Hürde blieben. ${ }^{25}$ Die beiden „Großparteien“ erhielten zusammen rund zwei Drittel der abgegebenen Stimmen (68,3 Prozent / +6,3) - weit entfernt von einer Stimmenkonzentration von deutlich über 80 und bis zu 90 Prozent bei den Wahlen der 1980/90er Jahre. Vor allem die Zugewinne der SPD (+2,0) auf 20,6 Prozent - ihr drittschlechtestes Ergebnis seit 1946 und zehn und mehr Prozentpunkte unter den Ergebnissen der Wahlen der 1970er bis 1990er Jahre - fielen mager aus in Anbetracht der Erwartungen, die die Oppositionsparteien und vor allem die Sozialdemokraten mit der Kandidatur Christian Udes verbanden.

Der Landtag setzt sich aus 180 Abgeordneten zusammen, von denen eine Hälfte in Einer-Stimmkreisen mit relativer Mehrheit, die andere über Wahlkreislisten gewählt wird. Im Vergleich zur übrigen Bundesrepublik weist das bayerische Wahlsystem, die „verbesserte Verhältniswahl“, dabei eine Reihe von Besonderheiten auf ${ }^{26}$ : (1) Die lose gebundene Liste gibt den Wählern die Möglichkeit zur Präferenzstimmgebung mit der Zweitstimme; (2) Erstund Zweitstimme werden beide bei der Stimmenverrechnung und Mandatszuteilung berücksichtigt; (3) die Stimmenübertragung erfolgt zwar auch nach dem Verfahren Hare/Niemeyer, allerdings nicht im gesamten Wahlgebiet, also landesweit/gesamtbayerisch, sondern ausschließlich auf Wahlkreisebene in sieben Wahlkreisen unterschiedlicher Größe, die den Regierungsbezirken entsprechen. ${ }^{27}$ Zusammen mit der landesweiten Sperrklausel, von der die Hauptwirkung ausgeht, führt dies zu beachtlichen Disproportionen von Stimmen und Mandaten; davon profitieren alle Parlamentsparteien, vor allem aber selbstverständlich die CSU am stärksten, die im Maximilianeum über 101 Abgeordnete verfügt - neun mehr als in der vorangegangenen Wahlperiode - und deutlich überrepräsentiert ist (siehe Tabelle 4).

Die Möglichkeit der Präferenzstimmgebung wird von den Wählern durchaus häufig genutzt, auch wenn die überwiegende Zahl der Stimmen sich auf die jeweiligen (Wahlkreis-) Listenführer konzentriert. Allerdings unterscheiden sich die parteiinternen Auswirkungen:

25 Die Alternative für Deutschland (AfD) trat zur Landtagswahl nicht an; bei der eine Woche später abgehaltenen Bundestagswahl erzielte sie landesweit in Bayern 4,3, bundesweit 4,7 Prozent der Zweitstimmen.

26 Zum Wahlsystem siehe Emil Hübner, Das bayerische Landtagswahlrecht. Grundzüge - Nutzung - Reformmodell, in: Reinhold L. Bocklet (Hrsg.), Das Regierungssystem des Freistaats Bayern, Band 2, München 1979, S. 279 - 294.

27 Aufgrund der Neueinteilung der Stimmkreise verloren die Oberpfalz und Oberfranken je ein Mandat pro Stimmkreis, die beide dem Regierungsbezirk beziehungsweise Wahlkreis Oberbayern zugeschlagen wurden. Die Zahl der 180 Mandate verteilt sich auf die Regierungsbezirke deshalb jetzt wie folgt: Oberbayern 60, Niederbayern 18, Oberpfalz 16, Oberfranken 16, Mittelfranken 24, Unterfranken 20, Schwaben 26 Mandate. In den Regierungsbezirken mit unter 20 Mandaten sind infolgedessen über fünf Prozent der Stimmen erforderlich, um dort an der Mandatsverteilung überhaupt beteiligt zu sein. 


\begin{tabular}{|l|c|c|c|c|c|c|}
\hline \multicolumn{7}{|c|}{ Tabelle 4: Amtliches Endergebnis der bayerischen Landtagswahl vom 15. September 2013 } \\
\hline \multirow{2}{*}{ Partei } & Gesamtstimmen 2013 & Gesamtstimmen 2008 & \multicolumn{2}{|c|}{ Mandate 2013 } \\
\cline { 2 - 7 } & absolut & in Prozent & absolut & in Prozent & absolut & in Prozent \\
\hline CSU & 5.636 .425 & 47,7 & 4.603 .960 & 43,4 & 101 & 56,1 \\
SPD & 2.437 .401 & 20,6 & 1.972 .437 & 18,6 & 42 & 23,3 \\
Grüne & 1.019 .373 & 8,6 & 999.111 & 9,4 & 18 & 10,0 \\
FW & 1.062 .553 & 9,0 & 1.085 .896 & 10,2 & 19 & 10,6 \\
FDP & 390.038 & 3,3 & 847.227 & 8,0 & - & - \\
Die Linke & 251.097 & 2,1 & 461.755 & 4,4 & - & - \\
Ödp & 239.425 & 2,0 & 212.200 & 2,0 & - & - \\
Sonstige & 785.552 & 6,6 & 429.689 & 4,1 & - & - \\
\hline Anmerkung: Fehlende Übereinstimmungen zu 100 Prozent sind rundungsbedingt. & \\
Quelle: Bayerisches Landesamt für Statistik und Datenverarbeitung, a.a.O. (Fn. 1). \\
\hline
\end{tabular}

Ihr Ausmaß hängt erstens ab von der höchst einseitigen Chance das Stimmkreis-Mandat zu gewinnen; mit Ausnahme eines der Münchener Stimmkreise, den die SPD-Bewerberin Ruth Waldmann holen konnte, fielen alle anderen 89 Stimmkreise an die CSU, die zusätzlich zwölf Listenmandate erzielte, während die Mandatsverteilung an die Oppositionsparteien über die Wahlkreislisten erfolgte. Die Auswirkungen hängen zweitens wegen der Addition von Erst- und Zweitstimme von der Stimmkreisgröße und der unterschiedlichen Zahl von Wahlberechtigen pro Stimmkreis ab, so dass Bewerber aus großen Stimmkreisen deutlich bessere Aussichten besitzen als ihre Mitbewerber auf der Wahlkreisliste aus Stimmkreisen mit weniger Wahlberechtigten. Und dennoch gehen von der Auswahlmöglichkeit durchaus beachtliche Wirkungen sowohl auf die innerparteilichen Karrierechancen als auch auf die Zusammensetzung der Landtagsfraktionen aus. Dies betrifft die Bewerber aller Parlamentsparteien, von denen viele gut platzierte Kandidaten vom Wähler abgestraft wurden, im Gesamtergebnis auf der Liste Rangplätze einbüßten oder gar das aufgrund des Listenplatzes als sicher angesehene Mandat nicht gewannen. Andere wurden vom Wähler präferiert, gewannen deutlich an Stimmen und Rangplätzen hinzu, in einer Reihe von Fällen bis hin zum Mandatsgewinn. Selbst für CSU-Kandidaten - wenn auch in geringerer Zahl lässt sich dies feststellen, unter anderem in den Wahlkreisen Oberbayern, Niederbayern, Unterfranken und Schwaben, in Oberbayern etwa auch für den nur auf der Liste kandidierenden vormaligen Wissenschaftsminister Thomas Goppel. ${ }^{28}$ Selbstverständlich betrifft es aber insbesondere die Zusammensetzung der drei Oppositionsfraktionen, bei denen auch eine Reihe von Spitzenpolitikern den Sprung in den Landtag gerade noch schaffte oder gar verfehlte. Auf den Listen der SPD gewannen das Mandat allein im Wahlkreis Oberbayern sechs weit hinter der dortigen SPD-Mandatszahl platzierte Bewerber; in Mittelfranken waren es zwei, in Schwaben ein Bewerber. Bei den Freien Wählern waren es im Wahlkreis Oberbayern zwei Kandidaten, während die Abgeordnete Ute Singer (alias die Sängerin

28 Thomas Goppel, weit hinten platziert, erreichte auf der Oberbayern-Liste die vierthöchste Zahl an Zweitstimmen nach Horst Seehofer, Ilse Aigner und Christine Haderthauer auf den Listenplätzen eins bis drei. 
Claudia Jung) trotz guter Platzierung ihr Mandat einbüßte. Bei den Grünen war die Wirkung des Stimmgebungsverfahrens der lose gebundenen Liste noch einschneidender, denn mit Therese Schopper und Martin Runge verfehlten die amtierenden Ko-Vorsitzenden von Partei beziehungsweise Fraktion und mit Susanna Tausendfreund und Eike Hallitzky weitere Spitzenkräfte den Sprung in den Landtag.

\subsection{Wählerverhalten nach Regionen}

Das landesweite Ergebnis überdeckt dabei die regionalen Unterschiede, die diesmal allerdings weniger ausgeprägt ausfielen als bei der Landtagswahl vor fünf Jahren. Hatte die CSU 2008 in allen Landesteilen, vor allem in den drei Regionen Altbayerns - in Ober- und Niederbayern sowie in der Oberpfalz -, knapp über beziehungsweise unter 20 Prozentpunkte gegenüber der Ausnahmewahl von 2003 eingebüßt, verbuchte sie diesmal in sechs der sieben Regierungsbezirke teilweise deutliche Stimmenzuwächse, in Altbayern ein Plus von 6,9 Punkten auf einen Gesamtstimmenanteil von 48,2 Prozent, während die Gewinne in Franken und Schwaben mit 2,1 und 2,3 Punkten um einiges hinter dem landesweiten Durchschnitt zurückblieben. Im protestantischen Mittelfranken erzielte die CSU mit 41,3 Prozent ihr landesweit schlechtestes Ergebnis, ein Minus von 0,7 Prozentpunkten (siehe Tabelle 5). Das Pendel schlug folglich deutlich in die Gegenrichtung aus, so dass sich die Abstände verringerten und sich die Differenzen in den regionalen CSU-Stimmenergebnissen mehr als ansatzweise wieder den altbekannten Mustern annäherten mit dem traditionellen Übergewicht Altbayerns und Schwabens gegenüber Nordbayern und vor allem der mittelfränkischen Nürnberger Region. Dies hängt sicherlich auch zusammen mit den veränderten Personalkonstellationen nach der Ablösung des Protestanten Günther Beckstein durch den katholischen Oberbayern Horst Seehofer im Amt des Ministerpräsidenten nach der letzten Wahl von 2008 und durch den Wechsel von Ilse Aigner von der Bundes- in die Landespolitik.

\begin{tabular}{|l|c|c|c|c|c|c|c|c|c|c|}
\hline Tabelle 5: Ergebnisse in den Regierungsbezirken und Veränderung gegenüber 2008 \\
\hline & \multicolumn{2}{|c|}{ CSU } & \multicolumn{2}{c|}{ SPD } & \multicolumn{2}{c|}{ Grüne } & \multicolumn{2}{c|}{ FW } & \multicolumn{2}{c|}{ FDP } \\
\cline { 2 - 10 } & $\begin{array}{c}\text { in } \\
\text { Prozent }\end{array}$ & $\begin{array}{c}\text { Diff. zu } \\
2008\end{array}$ & $\begin{array}{c}\text { in } \\
\text { Prozent }\end{array}$ & $\begin{array}{c}\text { Diff. zu } \\
2008\end{array}$ & $\begin{array}{c}\text { in } \\
\text { Prozent }\end{array}$ & $\begin{array}{c}\text { Diff. zu } \\
2008\end{array}$ & $\begin{array}{c}\text { in } \\
\text { Prozent }\end{array}$ & $\begin{array}{c}\text { Diff. zu } \\
2008\end{array}$ & $\begin{array}{c}\text { in } \\
\text { Prozent }\end{array}$ & $\begin{array}{c}\text { Diff. zu } \\
2008\end{array}$ \\
\hline $\begin{array}{l}\text { Wahlergebnis } \\
\text { (insgesamt) }\end{array}$ & 47,7 & $+4,3$ & 20,6 & $+2,0$ & 8,6 & $-0,8$ & 9,0 & $-1,2$ & 3,3 & $-4,7$ \\
\hline Bezirke & & & & & & & & & & \\
Oberbayern & 47,2 & $+7,9$ & 22,1 & $+2,8$ & 9,9 & $-3,2$ & 7,1 & $-1,6$ & 3,9 & $-6,1$ \\
Niederbayern & 50,4 & $+4,9$ & 14,0 & $-0,1$ & 5,9 & $+0,1$ & 14,4 & $+1,5$ & 3,6 & $-4,6$ \\
Oberpfalz & 49,9 & $+5,4$ & 19,5 & $+0,7$ & 5,7 & $+0,1$ & 12,4 & $-1,2$ & 1,9 & $-4,0$ \\
Oberfranken & 45,9 & $+1,2$ & 23,3 & $+2,1$ & 7,1 & $+0,4$ & 9,3 & $-1,4$ & 2,8 & $-3,8$ \\
Mittelfranken & 43,1 & $-0,7$ & 24,6 & $+2,0$ & 9,9 & $+1,2$ & 7,5 & $-1,8$ & 3,0 & $-3,2$ \\
Unterfranken & 50,1 & $+2,8$ & 19,5 & $+2,8$ & 8,9 & $+0,5$ & 8,2 & $-2,1$ & 2,9 & $-3,8$ \\
Schwaben & 49,4 & $+2,4$ & 17,2 & $+0,8$ & 8,6 & $\pm 0,0$ & 9,7 & $-0,8$ & 3,4 & $-4,5$ \\
\hline
\end{tabular}


Die Sozialdemokraten erzielten ihre allerdings bescheidenen besten Stimmenergebnisse mit 24,6 und 23,3 Prozent wiederum in Mittel- und Oberfranken, gefolgt von Oberbayern mit 22,1 Prozent, ihre schlechtesten Ergebnis wie in der Vergangenheit in Niederbayern, Schwaben und der Oberpfalz. Überdurchschnittlich erfolgreich war die SPD nur in Gebieten mit besonders hoher Bevölkerungsdichte; sie blieb allerdings selbst in München, Nürnberg und Augsburg nur zweiter Sieger hinter der CSU, die in Gebieten mit hoher Bevölkerungsdichte anders als 2008 wieder deutlich über 40 Prozent der Stimmen erhielt. ${ }^{29}$ Ihre besten Ergebnisse verbuchte die Union - wie zu erwarten - jedoch mit knapp unter 50 bei mittlerer und mit deutlich über 50 Prozent bei geringer Bevölkerungsdichte auf dem Lande. ${ }^{30}$

Über regionale und lokale Schwerpunkte verfügen auch die zwei „,kleinen“ Parlamentsparteien, Freie Wähler und Bündnis 90/Die Grünen. Sie sind im Vergleich zu der vorangegangenen Wahl allerdings weniger ausgeprägt. Dies gilt insbesondere für die Grünen, die in ihrem Kerngebiet Oberbayern 3,2 Prozentpunkte einbüßten und dort auf den Stand der Wahl von 2003 zurückfielen, während die Partei in den sechs anderen Regionen Stimmenund Prozentzuwächse verzeichnete, in Nordbayern deutlicher als im Süden des Freistaates. Schwache Ergebnisse erzielten die Grünen weiterhin im ländlichen Raum Niederbayerns und der Oberpfalz mit deutlich unterdurchschnittlichen Resultaten; überdurchschnittlich fielen die Ergebnisse in Mittel- und Unterfranken aus. Die Grünen sind auch weiterhin primär eine Partei (groß-)städtischer Wählerschaft mit einem Stimmenanteil in München von 12,1 Prozent, in Nürnberg von 10,7 und in Augsburg von 11,6 Prozent. Demgegenüber haben die Freien Wähler neuerlich überproportional stark in eher ländlich geprägten Regionen abgeschnitten. Mit Ausnahme von Niederbayern, der Heimatregion Hubert Aiwangers, wo sie sich auf 14,4 Prozent steigern konnten, mussten sie in allen Regionen prozentuale Verluste hinnehmen, selbst in der Oberpfalz, ihrer zweiten altbayerischen Hochburg, vor allem aber in Franken und Oberbayern, wo sie trotz der beachtlich gestiegenen Wahlbeteiligung auch in den absoluten Stimmenzahlen hinter ihre Ergebnisse von 2008 zurückfielen. Dies gilt insbesondere für die hoch verdichteten städtischen und großstädtischen Räume, wo die Freien Wähler nur auf 5,8 Prozent kamen, und in München, wo sie erneut unter der Fünf-Prozent-Hürde blieben. ${ }^{31}$ Freie Wähler und Grüne konkurrieren damit lokal um unterschiedliche Wählerschaften, die zudem sozialstrukturell deutliche Unterschiede aufweisen.

\subsection{Sozialstrukturelle Aspekte des Wählerverhaltens}

Den offenkundigen Asymmetrien in der bayerischen Politik, wie sie im Wählerverhalten 2013 wieder zum Ausdruck gekommen sind, sind langfristig strukturell wie kurzfristig situativ und konjunkturell begründet. So spielen traditionelle Milieus und Parteibindungen im Verhalten bayerischer Wähler noch immer eine deutlich größere Rolle als anderswo in der

29 Vgl. Forschungsgruppe Wahlen, a.a.O. (Fn.1), S. 58 ff. In München erhielt die SPD beispielsweise 32,1, in Nürnberg 29,7 und in Augsburg 25,9 Prozent der Stimmen, und liegt damit in München nur knapp um 4,6, in den beiden anderen Großstädten, um 9,7 und 15,9 Prozent hinter der CSU.

30 Vgl. ebenda.

31 Vgl. ebenda. 
Bundesrepublik. Die Wählerbindung an die CSU liegt, ausweislich der Zahlen der Forschungsgruppe Wahlen, bei circa 35 Prozent und damit rund zehn Punkte über der ihrer Schwesterpartei außerhalb Bayerns, ganz zu schweigen von der weit geringeren Kernklientel der SPD. ${ }^{32}$ Damit sind und bleiben in Bayern CSU-Stimmenergebnisse von rund 50 Prozent durchaus im Bereich des Möglichen. Dazu bedarf es allerdings besonders günstiger Umstände. Infolgedessen kam die Partei der magischen Zahl mit 47,7 und 49,3 Prozent bei den beiden Wahlen 2013 recht nahe.

In Anbetracht dieser Zahlen verwundert es nicht, dass die CSU in allen Wählergruppen am besten abgeschnitten hat und fast überall deutlich über 40 Prozent liegt. Dennoch ergeben sich in der sozialstrukturellen Zusammensetzung der CSU-Wählerschaft auch Differenzen; sie akzentuieren neuerlich die altbekannten Muster mit ihren typischen, teils spezifisch bayerischen Ausprägungen in Politischer Kultur und Wählerlandschaft. Dies gilt zum einen für die traditionelle Überrepräsentation in der katholischen Wählerschaft - immerhin über 60 Prozent aller Wahlberechtigten in Bayern - mit einem CSU-Stimmenanteil von 56 Prozent, der mit zunehmender Kirchenbindung bis auf 71 Prozent bei stark gebundenen Wählern ansteigt. Bei den protestantischen Wählern erzielt die CSU mit einem Anteil von 44 Prozent hingegen ein fast durchschnittliches Ergebnis und bei konfessionslosen Wählern wie zu erwarten - schneidet sie nur stark unterdurchschnittlich ab. ${ }^{33}$ Es zeigt sich des Weiteren bei Bildungs- wie Berufsstruktur der Wählerschaften, denn die CSU verbucht in der Arbeiterschaft erneut bessere Ergebnisse als bei Beamten und Angestellten und ist bei Wählern mit niederer und mittlerer Schulbildung deutlich erfolgreicher als bei Wählern mit gymnasialen oder akademischen Bildungsabschlüssen (siehe Tabelle 6). Zur Kernklientel der CSU gehören zudem Landwirte und Rentner. Bei den über 60-jährigen Wählern votierten circa 57 Prozent für die Union; am stärksten waren die Zuwächse gegenüber 2008 indessen in den Altersgruppen der 30- bis 59-Jährigen, wobei die Zugewinne bei den Frauen mit zwölf Prozentpunkten doppelt so stark ausfielen wie bei ihren männlichen Altersgenossen. ${ }^{34}$ Die CSU ist damit sozialstrukturell wieder sehr breit in der Wählerschaft verankert - anders als 2008, als ihr Volksparteicharakter aufgrund der überproportionalen Stimmeneinbußen in manchen Wählerschichten in Gefahr zu geraten schien.

Die Wählerschaft der SPD unterscheidet sich nicht nur wegen ihrer Größenordnung um 20 Prozent deutlich von jener der Union. Wenngleich die Differenzen in der Alters-, Bildungs- und Berufsstruktur der SPD-Wähler nicht sonderlich groß ausgeprägt sind, so fällt doch auf, erstens, dass die sozialdemokratische Wählerschaft deutlich protestantischer ist und dass die SPD mit 26 Prozent überdurchschnittlich von Konfessionslosen, aber nur von 16 Prozent der Katholiken gewählt wurde ${ }^{35}$; zweitens, dass die Sozialdemokraten bei Angestellten, Beamten und Rentnern besser abschneiden als in der Arbeiterschaft und sie - anders als aus der Vergangenheit gewohnt - bei den Jüngeren nur unterdurchschnittliche Anteile gewinnt, ihre Wählerschaft also altert (siehe Tabelle 7). Bemerkenswert ist des Weiteren, dass die SPD - anders als die CSU - bei Wählern mit Abitur und Hochschulbildung

32 Vgl. ebenda, S. 10.

33 Allerdings sind selbst in Bayern nur mehr elf Prozent der Gesamtwählerschaft stark konfessionell gebunden; insgesamt römisch-katholisch: 61, evangelisch: 22, ohne Konfession: 14 Prozent der Wahlberechtigten; vgl. Forschungsgruppe Wahlen, a.a.O. (Fn. 1), S. 37 f.; S. 54.

$34 \mathrm{Vgl}$. ebenda, S. $32 \mathrm{ff}$.

35 Vgl. ebenda. 


\begin{tabular}{|c|c|c|c|c|c|c|c|c|c|c|}
\hline \multicolumn{11}{|c|}{ Tabelle 6: Wablverhalten nach Bildung und Beruf sowie Veränderung gegenüber 2008} \\
\hline & \multicolumn{2}{|c|}{ CSU } & \multicolumn{2}{|c|}{ SPD } & \multicolumn{2}{|c|}{ Grüne } & \multicolumn{2}{|c|}{ FW } & \multicolumn{2}{|c|}{ FDP } \\
\hline & \begin{tabular}{|c|} 
in Pro- \\
zent
\end{tabular} & \begin{tabular}{|c|} 
Diff. zu \\
2008
\end{tabular} & \begin{tabular}{|c|} 
in Pro- \\
zent
\end{tabular} & \begin{tabular}{|c|} 
Diff. zu \\
2008
\end{tabular} & \begin{tabular}{|c|} 
in Pro- \\
zent
\end{tabular} & \begin{tabular}{|c|} 
Diff. zu \\
2008
\end{tabular} & $\begin{array}{c}\text { in Pro- } \\
\text { zent }\end{array}$ & $\begin{array}{c}\text { Diff. zu } \\
2008\end{array}$ & $\begin{array}{c}\text { in Pro- } \\
\text { zent }\end{array}$ & \begin{tabular}{|c} 
Diff. zu \\
2008
\end{tabular} \\
\hline $\begin{array}{l}\text { Wahlergebnis } \\
\text { (insgesamt) }\end{array}$ & 47,7 & $+4,3$ & 20,6 & $+2,0$ & 8,6 & $-0,8$ & 9,0 & $-1,2$ & 3,3 & $-4,7$ \\
\hline \multicolumn{11}{|l|}{ Bildung } \\
\hline $\begin{array}{l}\text { Hochschul- } \\
\text { abschluss }\end{array}$ & 36 & +4 & 24 & +4 & 19 & -1 & 8 & +1 & 5 & -6 \\
\hline Abitur & 42 & +4 & 20 & \pm 0 & 14 & \pm 0 & 9 & -2 & 3 & -7 \\
\hline Mittel & 51 & +7 & 19 & +2 & 7 & -1 & 9 & -3 & 3 & -5 \\
\hline Niedrig & 56 & +7 & 18 & \pm 0 & 3 & -1 & 8 & -3 & 2 & -4 \\
\hline \multicolumn{11}{|l|}{ Beruf } \\
\hline Arbeiter & 49 & +5 & 22 & +2 & 4 & -2 & 11 & -1 & 2 & -3 \\
\hline $\begin{array}{l}\text { Arbeiter/ } \\
\text { Gew. }\end{array}$ & 42 & +7 & 29 & +1 & 3 & \pm 0 & 10 & -1 & 2 & -2 \\
\hline Angestellte & 45 & +5 & 22 & +2 & 10 & -1 & 10 & -1 & 3 & -6 \\
\hline $\begin{array}{l}\text { Angestellte/ } \\
\text { Gew. }\end{array}$ & 35 & -3 & 30 & +6 & 9 & -1 & 8 & -1 & 2 & -2 \\
\hline Beamte & 42 & \pm 0 & 23 & +2 & 12 & +1 & 11 & \pm 0 & 5 & -1 \\
\hline Selbständige & 45 & +3 & 16 & +5 & 12 & -2 & 8 & -2 & 11 & -4 \\
\hline Landwirte & 67 & +1 & 5 & -2 & 2 & -2 & 15 & +6 & 4 & -1 \\
\hline Rentner & 55 & +3 & 25 & +3 & 4 & \pm 0 & 8 & \pm 0 & 4 & -3 \\
\hline Arbeitslose & 23 & -3 & 22 & +5 & 15 & +7 & 8 & -2 & 3 & -7 \\
\hline \multicolumn{11}{|c|}{$\begin{array}{l}\text { Anmerkung: Wahltagsbefragung der Forschungsgruppe Wahlen für das ZDF; Gesamtstimmenanteile in } \\
\text { Prozent; Veränderungen zu } 2008 \text { in Prozentpunkten; in der Kategorie Bildung ist mittel = Mittlere Rei- } \\
\text { fe, niedrig = Hauptschulabschluss; Gew. = gewerkschaftlich organisiert; laut Mikrozensus } 2011 \text { lag die } \\
\text { Erwerbsquote in Bayern bei 53,7 Prozent, davon waren Arbeiter: 25,6, Angestellte / Beamte: 61,2, Selb- } \\
\text { ständige: 13,2 Prozent. } \\
\text { Quelle: Forschungsgruppe Wahlen, a.a.O. (Fn. 1), S. 62, S. } 66 \text { ff. }\end{array}$} \\
\hline
\end{tabular}

höhere Stimmenanteile erreicht als bei solchen mit mittlerem oder niedrigem Bildungsniveau. Noch eindeutiger wird das Bild, wenn man nach dem Zusammenhang zwischen Alter und Bildungsgrad fragt. Während die CSU mit einem Anteil von 64 Prozent ihr bestes Ergebnis in der Gruppe der über 60-Jährigen mit Hauptschulabschluss erzielt, sind es bei der SPD überdurchschnittliche 26 Prozent in der Gruppe der über 60-jährigen Wähler mit Abitur oder Hochschulabschluss. Der typische SPD-Wähler kommt damit aus der Stadt, ist Beamter oder Angestellter und verfügt über einen qualifizierten Bildungsabschluss.

Die beiden kleinen Oppositionsparteien repräsentieren jeweils circa ein Zehntel der bayerischen Wähler, die sich in ihrer sozialen Zusammensetzung deutlich unterscheiden. Die offenkundigen Stadt-Land-Unterschiede korrelieren mit Differenzen in der Alters-, Bildungs-, Berufs- und Konfessionsstruktur ihrer Wählerschaften. Die Freien Wähler erzielen dabei in den verschiedenen Altersgruppen (mit Ausnahme der über 60-Jährigen), bei Männern und Frauen, aber auch in den verschiedenen Bildungsschichten und Berufsgruppen (und zwar auch unter den Arbeiterwählern) Resultate, die ihrem Gesamtstimmenergebnis nahe kommen, so dass sie in der Wählerschaft vergleichsweise breit vertreten sind und zu- 


\begin{tabular}{|l|c|c|c|c|c|c|}
\hline \multicolumn{7}{|c|}{ Tabelle 7: Stimmabgabe der Männer und Frauen nach Altersgruppen (in Prozent) } \\
\hline & CSU & SPD & Grüne & FW & FDP & Sonstige \\
\hline Wahlergebnis (insgesamt) & 47,7 & 20,6 & 8,6 & 9,0 & 3,3 & 10,8 \\
\hline Männer & & & & & & \\
18 bis 29 Jahre & 41 & 17 & 9 & 9 & 4 & 21 \\
30 bis 44 Jahre & 47 & 16 & 9 & 9 & 4 & 14 \\
45 bis 59 Jahre & 44 & 21 & 9 & 10 & 4 & 12 \\
über 60 Jahre & 56 & 24 & 4 & 7 & 5 & 5 \\
\hline Frauen & & & & & & \\
18 bis 29 Jahre & 40 & 20 & 12 & 9 & 2 & 17 \\
30 bis 44 Jahre & 45 & 18 & 12 & 10 & 3 & 13 \\
45 bis 59 Jahre & 43 & 22 & 13 & 10 & 2 & 10 \\
über 60 Jahre & 57 & 24 & 4 & 8 & 3 & 5 \\
\hline Männer und Frauen & & & & & & \\
18 bis 29 Jahre & 40 & 19 & 10 & 9 & 3 & 19 \\
30 bis 44 Jahre & 46 & 17 & 11 & 10 & 4 & 13 \\
45 bis 59 Jahre & 44 & 22 & 10 & 11 & 3 & 11 \\
über 60 Jahre & 57 & 23 & 4 & 7 & 5 & 5 \\
\hline Anmerkung: Wahltagbefragung der Forschungsgruppe Wahlen für das ZDF. & & \\
Quelle: Forschungsgruppe Wahlen, a.a.O. (Fn. 1), S. 64 f. & \multicolumn{5}{|l}{} \\
\hline
\end{tabular}

dem auf die überdurchschnittliche Unterstützung ländlicher Wähler, insbesondere der selbständigen Landwirte bauen können (siehe Tabelle 6). Anders als die einseitig wirtschaftsliberale, mittelständisch-städtische FDP sind die Freien Wähler damit sozialstrukturell und milieumäßig in der bayerischen Wählerschaft solide und wohl auch mittelfristig stabil verankert.

Die sozialstrukturelle Basis der grünen Wählerschaft hat sich nicht sonderlich verändert. Zwar sind die Grünen längst nicht mehr so attraktiv für Erst- und Jungwähler wie in der Vergangenheit; sie erzielen schon seit geraumer Zeit auch in den Altersgruppen der 30- bis 60-Jährigen durchschnittliche Ergebnisse und sind allein in der Generation der Älteren mit unter fünf Prozent deutlich unterrepräsentiert. Auch die grüne Wählerschaft ist wie die Partei selbst in die Jahre gekommen. Sie altern beide und sind insofern ein Pendant der (auch in Bayern) alternden Gesellschaft. Wie in den vorangegangenen Wahlen erzielen die Grünen ihre besten Ergebnisse bei den Hochgebildeten, und die grüne Wählerschaft ist weiblicher (siehe Tabelle 6 und 7). Während Arbeiter, Rentner, aber auch Landwirte deutlich unterrepräsentiert sind, machen Angestellte und Beamte - insgesamt nach dem Mikrozensus von 2011 über 60 Prozent der Erwerbstätigen in Bayern - weiterhin den Kern der grünen Wählerschaft aus, gefolgt in beachtlichem Umfang von Selbständigen (siehe Tabelle 6). Allerdings unterscheiden sich mit den differenzierten, ja gegensätzlichen Interessenlagen unter Angestellten und Beamten auch deren politische Präferenzen: Die grünen Wähler sind vornehmlich in Berufen sozialer wie kultureller Dienstleistungen und der Wissensgesellschaft erwerbstätig; sie entstammen der "sozialen und kulturellen Dienstklasse“(in der Terminologie der Soziologen John Goldthorpe, Walter Müller und anderer). Sie bilden auch 
in der bayerischen Wählerschaft den „klassenstrukturellen Kern“ der Grünen, während die Angehörigen der „administrativen Dienstklasse“, also unter anderem die Manager und weitere Beschäftigte mit Leitungsfunktionen, insbesondere in der Privatwirtschaft, beruflich, kulturell wie auch in den Parteipräferenzen den Gegenpol ausmachen. ${ }^{36}$ Anders als manche Kommentatoren in Anbetracht des gemeinsamen hohen Bildungsniveaus und vielfach auch überdurchschnittlicher Einkommen glauben annehmen zu können, bestehen die gegensätzlichen Interessenlagen in den Dienstklassen von Angestellten und Beamten sowie die milieumäßigen Spaltungen fort; sie dürften in Bayern im Übrigen nicht weniger ausgeprägt sein als in anderen Regionen der Bundesrepublik. Dies ist durchaus von partei- wie koalitionsstrategischer Bedeutung.

Die Wähler sind mobiler geworden, und die Zahl der Wechselwähler steigt auch in Bayern. Allerdings ist die Richtung weiterhin in beachtlichem Maße strukturell mitbestimmt. Sie folgt gerade in Bayern unverändert (sozial-moralischen) Milieubindungen, wie locker oder auch nur mehr latent sie vorhanden sein mögen, und damit einhergehenden politischideologischen Grundorientierungen, wodurch die Wechselmöglichkeiten richtungsmäßig begrenzt sind. Die Wählerwanderungen der letzten drei Landtagswahlen seit 2003 belegen dies eindrucksvoll. Die dramatischen Verluste der CSU von 17,3 Prozentpunkten bei der Wahl 2008 resultierten vornehmlich aus Abwanderung in die Nichtwahl, durch den Generationenwechsel und lagerintern durch Wechsel zu den Freien Wählern und zur FDP, jedoch nur zu einem Viertel aus lagerübergreifendem Wechsel. ${ }^{37}$ Die Stimmengewinne der CSU 2013 speisen sich aus denselben Quellen: aus dem Lager der Nichtwähler (im Saldo 300.000), aus Zuwanderung nach Bayern (150.000) sowie aus dem Wechsel vormaliger FDP- (110.000), aber auch Freier Wähler (50.000). ${ }^{38}$ Auch die SPD profitiert, wenn auch weit weniger, von der deutlich gestiegenen Wahlbeteiligung, aus dem Zuzug von Wählern nach Bayern und nur in vergleichsweise geringem Maße aus Zuwanderung von Wechselwählern, primär von Grünen und Linkspartei. Im Saldo ermittelte Infratest dimap SPDGewinne von etwa 270.000 Stimmen, davon 110.000 durch die Mobilisierung von Nichtwählern, 120.000 durch Parteiwechsel. Dabei wanderten nur wenige vormalige Unions- oder FDP-Wähler zu den Sozialdemokraten, im Saldo etwa jeweils 20.000, so dass sich die Erwartung auf lagerübergreifende Wechselwähler, die man mit der Spitzenkandidatur Udes verband, nicht erfüllte. ${ }^{39}$ Nicht nur die „ungleiche“ Kandidatenalternative Seehofer / Ude spielte dabei - wie geschildert - eine Rolle. Von nicht unerheblicher Bedeutung war zudem der (auch, aber nicht nur zeitlich durch die Wahltermine gegebene) enge Zusammenhang von Bundes- und Landespolitik.

36 Siehe Walter Müller / Markus Klein, Die Klassenbasis in der Parteipräferenz des deutschen Wählers. Erosion oder Wandel?, in: Rüdiger Schmitt-Beck (Hrsg.), Wählen in Deutschland, PVS Sonderheft 45, Baden-Baden 2012, S. 85 - 110; vgl. Rainer-Olaf Schultze, Trends der Wahlforschung, in: Uwe Andersen (Hrsg.), Die Wahl des Deutschen Bundestages, Schwalbach am Taunus 2013, S. $107-137$.

37 Die Darstellung orientiert sich an den Daten der Infratest dimap-Wanderungsbilanzen; vgl. Infratest dimap, Wahlreport. Landtagswahl in Bayern 2008, Berlin 2008; Infratest dimap, a.a.O. (Fn. 1), passim; für 2008 vgl. auch Rainer-Olaf Schultze / Jan Grasnick, a.a.O. (Fn. 2), S. 48 ff.

38 Vgl. Infratest dimap, a.a.O. (Fn. 1), S. 11 ff.

39 Vgl. ebenda, passim. 


\subsection{Wählerverhalten im Mehrebenensystem}

Den Wahlen zu den Parlamenten und Ämtern der vier politischen Systemebenen, von der Kommune bis zum Europäischen Parlament, kommt selbstverständlich unterschiedliches Gewicht zu. Die Wähler haben dafür auch ein gutes Gespür, was man nicht zuletzt an den starken Differenzen in der Höhe der Wahlbeteiligung ablesen kann. ${ }^{40}$ Der Einfluss der Bundespolitik auf die Wahlen der anderen Arenen ist sicherlich beachtlich. Die Wahlforschung ist sich auch weithin darin einig, dass die bundespolitische Durchdringung der Landtagswahlen weiter gewachsen ist ${ }^{41}$, wobei die Detailbegründungen - unter anderem die Thesen vom Wahlzyklus, vom Popularitätsgrad der Bundesregierung, vom Oppositionseffekt und moderierendem Wählen bei den „Nebenwahlen“ - allerdings unterschiedlich ausfallen. Doch rechtfertigt dies, von „Haupt- und Nebenwahlen“ zu sprechen? Denn die Einflüsse können ja durchaus wechselseitig sein und stellen keineswegs eine Einbahnstraße dar, wie es die These von den Haupt- und Nebenwahlen zumindest indirekt unterstellt. Oscar W. Gabriel und Everhard Holtmann versehen den Titel ihres Beitrages „Ober sticht Unter? Zum Einfluss der Bundespolitik auf Landtagswahlen“ bewusst mit einem Fragezeichen; sie sprechen zu Recht von „gesamtsystemarem Wahlverhalten“ und damit aufgrund der komplexen Gemengelage von der Notwendigkeit einer gesamtsystemaren Betrachtung. ${ }^{42}$

Am bayerischen Wählerverhalten kann man beides nachdrücklich zeigen: die bundespolitische Durchdringung, die als Folge der Doppelrolle der CSU als bayerischer Bundespartei besonders ausgeprägt ist ${ }^{43}$, aber eben auch die wechselseitigen Einflüsse der unterschiedlichen Wahlebenen aufeinander. Sowohl 2008/2009 als auch 2013/2014 sind die Wähler in Bayern auf allen vier Wahlebenen zur Urne gerufen worden, in relativ kurzen Abständen, aber auch in unterschiedlicher Abfolge: Das Doppelwahljahr 2008/2009 begann im März 2008 mit der Kommunalwahl, gefolgt von der Landtagswahl im September; an die EPWahl im Juni 2009 schloss sich im September die Bundestagswahl an. Demgegenüber startete das Doppelwahljahr 2013/2014 fast zeitgleich mit Landtags- und Bundestagswahl im September, auf die sechs Monate später im März 2014 die Kommunalwahl und Ende Mai die EP-Wahl folgten.

Befragt zu den Landtagswahlen von 2008 und 2013, waren die bayerischen Wähler in beiden Fällen zu etwa zwei Dritteln der Meinung, dass für ihre Wahlentscheidung die Landespolitik wichtiger gewesen sei als die Bundespolitik. ${ }^{44}$ Nur bei den Wahlen der Jahre

40 In Bayern schwankte die Wahlbeteiligung zwischen 42,3 (Wahl zum Europäischen Parlament 2009), 59,5 (Kommunalwahl 2008), 57,9 beziehungsweise 63,6 Prozent (Landtagswahlen 2008 und 2013) und 71,6 beziehungsweise 70,6 Prozent (Bundestagswahlen 2009 und 2013).

41 Siehe die zahlreichen Beiträge in Heft 3/2007 der ZParl; sodann Kerstin Völkl, Reine Landtagswahlen oder regionale Bundestagswahlen? Eine Untersuchung des Abstimmungsverhaltens bei Landtagswahlen 1990 - 2006, Baden-Baden 2009; dies. / Kai-Uwe Schnapp / Everhard Holtmann I Oscar W. Gabriel, Wähler und Landtagswahlen in der Bundesrepublik Deutschland, Baden-Baden 2008.

42 Vgl. Oscar W. Gabriel/ Everhard Holtmann, Ober sticht Unter? Zum Einfluss der Bundespolitik auf Landtagswahlen: Kontext, theoretischer Rahmen und Analysemodelle, in: ZParl, 38. Jg. (2007), H. 3, S. $445-462$.

43 Vgl. Harald Schoen, a.a.O. (Fn. 2), passim.

44 Die Forschungsgruppe Wahlen ermittelte im Verhältnis Landes- zu Bundespolitik 2008 eine Relation von 68 zu 28 Prozent, 2013 von 65 zu 32 Prozent; vgl. für 2008 Forschungsgruppe Wah- 
2008 und 2009 lagen die Wähler mit ihrer Einschätzung richtig. Die Landespolitik dominierte das Wählerverhalten - und zwar bei den Wahlen aller vier Ebenen. Die Wähler verpassten der CSU-Alleinregierung unter Edmund Stoiber und seinen Amtsnachfolgern Günther Beckstein und Erwin Huber - 2003 ausgestattet mit einer Zweidrittel-Mandatsmehrheit im Bayerischen Landtag - aus landespolitischem Verdruss mehr als einen Denkzettel. ${ }^{45}$ Sie wurde abgestraft wegen ihres von technokratischer Arroganz und verschiedenen Skandalen charakterisierten Politikstils und zugleich wegen zahlreicher unpopulärer landespolitischer Weichenstellungen, unter anderem der G-8-Schulpolitik, wie einer Reihe kommunalpolitisch wirksamer strittiger Entscheidungen auf dem Felde regionaler Strukturpolitik. In Anbetracht dieser Grundstimmung, auch in weiten Kreisen traditioneller CSU-Anhänger, bildeten die Kommunalwahlen im März 2008 den Vorboten für die Wählerentscheidung bei den sich anschließenden überregionalen Wahlen. Bei einer Wahlbeteiligung von unter 60 Prozent kam die CSU landesweit insgesamt nur auf rund 40 Prozent der Stimmen und verlor hauptsächlich an die kleinen Parteien, allen voran die Grünen und die Freien Wähler. Letztere konnten zusammen 19 Prozent der Stimmen auf sich vereinigen. Die Stimmenanteile der CSU unterschieden sich bei den Folgewahlen nicht sonderlich. Selbst bei der 18 Monate später abgehaltenen Bundestagswahl vom September 2009 erreichte sie mit 2.830.238 Zweitstimmen und 42,5 Prozent ein vergleichbares Ergebnis. Lediglich bei der EP-Wahl im Juni 2009 erzielte die Partei bei allerdings nur 1.896.762 Stimmen mit 48,1 Prozent einen deutlich höheren Stimmenanteil. Dies ist erstens auf die mit 42,3 Prozent extrem niedrige Wahlbeteiligung zurückzuführen, die sich gemäß dem so genannten Standardmodell politischer Partizipation ${ }^{46}$ zulasten der linken und zugunsten der bürgerlichen Parteien und damit nicht zuletzt der CSU auswirkt. Zweitens ist es eine Konsequenz des schlechten Abschneidens der Freien Wähler, die bislang nicht (und wohl auch zu Recht) als überregional-nationaler oder gar europäischer Partei-Akteur wahrgenommen werden. Sieht man von der EP-Wahl ab, war das Wählerverhalten im Doppelwahljahr 2008/2009 bei aller wechselseitigen Durchdringung primär von der Landespolitik geprägt. Von ihr gingen zunächst auf kommunaler Ebene beachtliche Veränderungen in den Mehrheits- und Regierungsverhältnissen aus. Dies untermauerte die ohnehin in Gang befindlichen Prozesse der Pluralisierung der politischen Landschaft und des Parteienwettbewerbs in Bayern.

Im Doppelwahljahr 2013/2014 stellte sich die Lage völlig anders dar. Obwohl die Wähler der Überzeugung waren, ihre Entscheidung aus landespolitischen Gründen getroffen zu haben, stand die Landtagswahl ganz eindeutig im Zeichen der Bundespolitik (siehe Tabelle 8). Es ging Seehofer und der CSU um die Wiedergewinnung der absoluten Mandatsmehrheit im Lande mittels einer bundespolitisch zentrierten Wahlstrategie. Sie bauten auf die Vorteile der besonderen Doppelrolle der CSU, spielten - entgegen aller öffentlichen Rhetorik vom Primat bayerischer Politik - spätestens seit der CDU-Niederlage bei der Landtagswahl in Nordrhein-Westfalen vom Mai 2012 die Karte eines Verbundwahlkampfes auf der Grundlage ihrer bundespolitischen Agenda. ${ }^{47}$ Zwar spielte im Wahlkampf auch Landespolitik eine Rolle, doch selbst im Bayern-Plan der CSU vom April 2013, dem Wahl- und

len, Wahl in Bayern. Eine Analyse der Landtagswahl vom 28. September 2008, Berichte Nr. 132, Mannheim 2008, passim; für 2013 Forschungsgruppe Wahlen, a.a.O. (Fn. 1), S. 11.

$45 \mathrm{Vgl}$. Rainer-Olaf Schultze / Jan Grasnick, a.a.O. (Fn. 2), passim.

46 Vgl. Rainer-Olaf Schultze, a.a.O. (Fn. 36), S. $120 \mathrm{ff}$.

47 Siehe im Einzelnen Gerhard Hirscher, a.a.O. (Fn. 3), S. 173 ff. 


\begin{tabular}{|l|c|c|c|c|}
\hline \multirow{3}{*}{ Tabelle 8: Landtags- und Bundestagswahlergebnis September 2013 im Vergleich } \\
\hline \multirow{3}{*}{ Partei } & \multicolumn{2}{|c|}{ Zweitstimmen Landtagswahl } & \multicolumn{2}{|c|}{ Zweitstimmen Bundestagswahl } \\
& 15. September 2013 & 22. September 2013 \\
\cline { 2 - 5 } & absolut & in Prozent & absolut & in Prozent \\
\hline CSU & 2.882 .169 & $48,9(+4,6)$ & 3.243 .569 & $49,3(+6,7)$ \\
SPD & 1.228 .957 & $20,8(+2,8)$ & 1.314 .009 & $20,0(+3,1)$ \\
Grüne & 497.056 & $8,4(-1,4)$ & 552.818 & $8,4(-2,4)$ \\
FW & 481.852 & $8,2(-1,6)$ & 180.649 & $2,7(+2,7)$ \\
FDP & 194.118 & $3,3(-4,6)$ & 334.158 & $5,1(-9,6)$ \\
Die Linke & 123.008 & $2,1(-2,2)$ & 248.920 & $3,8(-2,7)$ \\
AfD & - & - & 283.570 & $4,3(+4,3)$ \\
Sonstige & 450.655 & $8,3(-0,1)$ & 429.689 & $6,3(-2,4)$ \\
\hline Wahlbeteiligung & \multicolumn{5}{|c|}{$63,6(+5,7)$} & $70,0(-1,6)$ \\
\hline Anmerkung: Fehlende Übereinstimmungen zu 100 Prozent sind rundungsbedingt. \\
Quelle: Bayerisches Landesamt für Statistik und Datenverarbeitung, a.a.O. (Fn. 1). \\
\hline
\end{tabular}

Regierungsprogramm für die kommende Wahlperiode, standen solche Themen im Mittelpunkt, die auf der Entscheidungsebene der Bundespolitik angesiedelt sind: wirtschaftspolitisch unter anderem Haushaltskonsolidierung ohne Steuererhöhungen, Vollbeschäftigung und gesetzlicher Mindestlohn, Reform des Länderfinanzausgleichs mittels Klage vor dem Bundesverfassungsgericht; sozial- und familienpolitisch unter anderem Erhalt des Ehegattensplitting, Erhöhung des Kindergeldes, Betreuungsgeld und Mütterrente; institutionenpolitisch unter anderem Einführung von Volksabstimmungen in Fragen der EU-Verfassungspolitik; sodann insbesondere die Einführung der PKW-Maut für Ausländer. ${ }^{48}$ Seehofer und der CSU gelang es jedenfalls mit ihrem Verbundwahlkampf, die Agenda zu bestimmen. Damit liefen nicht nur die landespolitisch zentrierten Wahlstrategien der drei Oppositionsparteien weithin ins Leere. Die von der CSU ins Zentrum gerückten bundespolitischen Themen trafen nämlich auch die Erwartungshaltung und die konkreten Einstellungen der Mehrheit der bayerischen Wähler. So ermittelte Infratest dimap, dass 66 Prozent für die Einführung der PKW-Maut seien und über 70 Prozent für die Neuverhandlung des Länderfinanzausgleichs. Laut Forschungsgruppe Wahlen hielten etwa die Hälfte aller Wähler und 61 Prozent der CSU-Wähler die Einführung des Betreuungsgeldes für richtig. ${ }^{49}$ Wahlkampf und Wählerentscheidungen des Jahres 2013 waren damit primär bundespolitisch bestimmt. Allerdings war vom Trend der starken bundespolitischen Durchdringung bei der nur sechs Monate später im März 2014 abgehaltenen Kommunalwahl nicht mehr viel zu spüren: Die CSU erzielte wie schon 2008 landesweit nur circa 40 Prozent der Stimmen, nicht zuletzt entgegen den Erwartungen Seehofers und der CSU, die wohl vom fortdauernden Bundestrend ausgegangen waren und sich vom Ergebnis tief ent-

48 Zum Regierungsprogramm der CSU, dem Bayern-Plan 2013 bis 2018 siehe http://csu.de/programm/csu_regierungsprogramm_2013-2018_der_bayernplan/ (Abruf am 9. Mai 2014); vgl. Gerhard Hirscher, a.a.O. (Fn. 3), S. 195.

49 Vgl. Infratest dimap, a.a.O. (Fn. 1), S. 17 ff.; Forschungsgruppe Wahlen, a.a.O. (Fn .1), S. 21 ff. 
täuscht zeigten. ${ }^{50}$ Das bayerische Kommunalwahlergebnis 2014 ist jedenfalls ein weiteres deutliches Indiz gegen die zyklustheoretische Annahme, dass „Nebenwahl“-Ergebnisse umso weniger beziehungsweise umso stärker vom Bundestagswahlergebnis, insbesondere dem der führenden Bonner/Berliner Regierungspartei, abweichen, je geringer beziehungsweise größer der zeitliche Abstand vom Bundestagswahltermin ist.

Im Bezug auf die geforderte gesamtsystemare, die Wahlebenen übergreifende wie differenzierende Betrachtungsweise legen die geschilderten bayerischen Erfahrungen folgende Schlussfolgerungen nahe:

(1) Von wesentlicher Bedeutung sind die insgesamt rückläufigen, aber eben auch ebenenspezifisch stark variierenden Wahlbeteiligungsraten. Deren Einfluss wird häufig unterschätzt, zumal sie machtpolitisch nicht neutral sind und sich die soziale Asymmetrie in der politischen Beteiligung, gerade durch den epochalen Rückgang der konventionellen Partizipation bei Wahlen, verstärkt hat.

(2) Ebenenspezifische Varianzen und gegenläufige Einflüsse können sich aufgrund regional unterschiedlicher sozialstruktureller Bedingungen, Konfliktmuster und sozial-moralischer Milieubildungen ergeben, zumal wenn diese unterschiedliche Parteiensystem- und Wettbewerbskonstellationen zur Folge haben. Die bayerischen Wählerentscheidungen sind dafür unverändert ein anschauliches Bespiel.

(3) Die ebenenübergreifende Durchdringung der Wählerentscheidung ist keine Einbahnstraße, wie gemeinhin angenommen, sondern wechselseitig und vom Kontext abhängig. Der bundespolitische Einfluss auf die Landtagswahlen kann dabei durchaus überlagert oder gar in sein Gegenteil verkehrt werden. Wird er wirksam, spielt dabei weniger der zyklustheoretisch begründete zeitliche Abstand vom Bundestagswahltermin eine Rolle, sondern wohl eher - wie Simone Burkhart gezeigt hat - die (Un-)Popularität der jeweiligen Bundesregierung oder - wie die bayerischen Erfahrungen zeigen - von den Wählerurteilen über Bundespolitiker und/oder bundespolitisch agierende Landespolitiker. ${ }^{51}$ Besondere Bedeutung kommt dabei der Fähigkeit zu, die Deutungshoheit in der Wahlauseinandersetzung zu erlangen. Auch dies belegen die gegenläufigen Einflussrichtungen der jeweiligen bayerischen Doppelwahljahre auf eindrucksvolle Art.

\section{Regierungsbildung und Oppositionsformierung}

Mit der Regierungsbildung ließ sich der Ministerpräsident und Parteivorsitzende bis nach der Bundestagswahl Zeit. Vorgestellt wurde das Kabinett am 10. Oktober 2013. Ihm gehören außer dem Regierungschef elf Minister (davon fünf Frauen) und sechs Staatssekretäre mit Kabinettsrang an (siehe Tabelle 9). In Struktur und personeller Zusammensetzung trägt es ganz eindeutig die Handschrift Horst Seehofers. Seine Regierungsmannschaft ist personell von Macht-Balancierung, Rotation und innerparteilichem Regionalproporz charakterisiert,

50 Vgl. Mike Szymanski, Das Ende der CSU-Sause, in: SZ vom 18. März 2014, S. 32.

51 Siehe Simone Burkhart, Parteipolitikverflechtung. Über den Einfluss der Bundespolitik auf die Landtagswahlentscheidungen von 1976 bis 2002, in: PVS, 46. Jg. (2005), H. 1, S. $14-38$; Harald Schoen, a.a.O. (Fn. 2), passim; vgl. Kai-Uwe Schnapp, Landtagswahlen und Bundespolitik: Immer noch eine offene Frage? Neue Antworten im Ländervergleich auf Aggregatdatenbasis, in: ZParl, 38. Jg. (2007), H. 3, S. $464-480$. 


\begin{tabular}{|l|l|}
\hline Tabelle 9: Das Kabinett nach der bayerischen Landtagswabl vom 15. September 2013 \\
\hline Ministerpräsident & Horst Seehofer \\
\hline $\begin{array}{l}\text { Leiterin der Staatskanzlei und Staatsministerin für Bundesange- } \\
\text { legenheiten }\end{array}$ & Christine Haderthauer \\
\hline $\begin{array}{l}\text { Stellvertreterin des Ministerpräsidenten und Staatsministerin für } \\
\text { Wirtschaft, Medien, Energie, Technologie }\end{array}$ & Ilse Aigner \\
\hline Staatsminister der Finanzen, für Landesentwicklung und Heimat & Markus Söder \\
\hline Staatsminister des Innern, Bau, Verkehr & Joachim Herrmann \\
\hline Staatsminister für Justiz & Winfried Brausback \\
\hline Staatsminister für Bildung, Kultus, Wissenschaft und Kunst & Ludwig Spaenle \\
\hline Staatsministerin für Arbeit, Soziales, Familie und Integration & Emilia Müller \\
\hline Staatsministerin für Gesundheit und Pflege & Melanie Huml \\
\hline Staatsminister für Ernährung, Landwirtschaft und Forsten & Helmut Brunner \\
\hline Staatsminister für Umwelt und Verbraucherschutz & Marcel Huber \\
\hline Staatsministerin für Europaangelegenheiten & Beate Merk \\
\hline Staatssekretär im Innenministerium & Gerhard Eck \\
\hline Staatssekretäre im Kultus- und Wissenschaftsministerium & Georg Eisenreich / Bernd Sibler \\
\hline Staatssekretäre im Finanz-, Heimatministerium & $\begin{array}{l}\text { Albert Füracker / } \\
\text { Johannes Hintersberger }\end{array}$ \\
\hline Staatssekretär im Wirtschaftsministerium & Franz Pschierer \\
\hline Quelle: Eigene Zusammenstellung. & \\
\hline
\end{tabular}

sachpolitisch vom weitreichenden Neuzuschnitt der Ministerien. Neu in der Ministerriege sind die aus Oberfranken stammende Melanie Huml als Gesundsheitsministerin, vormals dort Staatssekretärin, und der Staatsrechtler Winfried Brausback, der Beate Merk als Justizminister ablöste, die ins wenig bedeutsame Ministerium für Europaangelegenheiten wechselte. Ihre Ämter behielten fünf Kabinettsmitglieder: der Landwirtschaftsminister Helmut Brunner sowie Joachim Herrmann, Marcel Huber, Ludwig Spaenle und Markus Söder, deren Aufgabenbereiche aber deutlich vergrößert und aufgewertet wurden. Umweltminister $H u$ ber ist auch für den Verbraucherschutz zuständig, Innenminister Herrmann zusätzlich für die Verkehrspolitik, während Spaenle neben dem Kultus- auch das Wissenschaftsministerium verantwortet. Zu den Schwergewichten im Kabinett gehören zweifellos Markus Söder, weiterhin Finanzminister und zugleich Minister für Landesentwicklung und Heimat, die in die Landespolitik gewechselte, oberbayerische CSU-Bezirksvorsitzende Ilse Aigner als Stellvertretende Ministerpräsidentin und als Wirtschaftsministerin auch zuständig für die Politikfelder Energie und Technologie sowie Christine Haderthauer, die Seehofer sich als Leiterin in die Staatskanzlei holte. Von ihr übernahm Emilia Müller das Arbeits- und Sozialministerium. Seehofer hat damit personell und sachlich die Macht im Kabinett außerordentlich geschickt entlang einer Strategie des Divide et Impera ausbalanciert, in der Hoffnung, dass sich die vier Superminister, also die beiden Franken Herrmann und Söder sowie die Oberbayern Aigner und Spaenle, außerdem die gleichfalls dem oberbayerischen Bezirksverband angehörende Haderthauer, in ihren Leistungen wechselseitig $\mathrm{zu}$ übertreffen versuchen, gleichzeitig aber auch in ihrer machtpolitischen Konkurrenz neutralisieren.

Den Fraktionsvorsitz der CSU übernahm Thomas Kreuzer, der nicht nur über langjährige Parlamentserfahrung verfügt und von 2003 bis 2011 als einer der stellvertretenden Frak- 
tionsvorsitzenden amtierte, sondern ab November 2011 unter Seehofer als Leiter der Staatskanzlei zum Koordinator der Regierungspolitik avancierte und das Vertrauen des Ministerpräsidenten besitzt. Als Fraktionsvorsitzender der SPD wurde Markus Rinderspacher bestätigt, der den langjährigen Vorsitzenden und Spitzenkandidaten Franz Maget im Oktober 2009 abgelöst hatte. Während die Führungspositionen in Partei und Fraktion der bayerischen Sozialdemokraten trotz der offensichtlichen Wahlniederlagen weithin unverändert blieben, löste das unbefriedigende Abschneiden bei den Grünen in beiden Doppelspitzen Veränderungen aus. Zur Ko-Vorsitzenden der Partei neben Dieter Janecek wurde als Nachfolgerin von Therese Schopper die Landshuter Lokalpolitikerin Sigi Hagl gewählt; an die Seite der weiter amtierenden Fraktionsvorsitzenden Margarete Bause tritt Ludwig Hermann als Ko-Vorsitzender. Bei den Freien Wählern bleiben Partei- und Fraktionsvorsitz in der Hand Hubert Aiwangers vereint.

In seiner Regierungserklärung „Bayern. Die Zukunft“ vom 12. November $2013^{52}$ benannte Horst Seehofer neben der Fortführung einer soliden Finanzpolitik vier große Zukunftsaufgaben, denen sich Regierung und Fraktion zu stellen haben: (1) die zunehmende Internationalisierung - oder: „Was müssen wir tun, um dauerhaft an der Spitze zu bleiben?“; (2) die digitale Revolution - oder: „Was müssen wir tun, um mit einem digitalen Aufbruch in die Weltspitze vorzustoßen?“; (3) die demografische Entwicklung - oder: „Was müssen wir tun, damit der demografische Wandel ein Gewinn wird?"; (4) die kulturelle Auswirkung oder: „Was müssen wir tun, damit Bayern für die Menschen lebens- und liebenswert bleibt?“. Programmatisch knüpfte Seehofer damit deutlich an die Politik der Ära Stoiber an: Es geht zum einen um Haushaltskonsolidierung, konkret um die schrittweise Schuldentilgung mit der Zielperspektive, im Jahr 2030 vollständig schuldenfrei zu sein. Und es geht zweitens um eine umfassend angelegte Politik technologischer Innovation bei gleichzeitiger Bewahrung der bayerischen wie regionalen landsmannschaftlichen Traditionen. Im Politikstil allerdings setzte Seehofer in der Regierungserklärung deutlich andere Akzente. Er versprach eine Politik des Dialogs und eine „Koalition mit den Bürgerinnen und Bürgern“, was seinem eigenen Handlungsstil entspricht, den Bürgern „aufs Maul zu schauen“, deren Stimmungen aufzugreifen und politisch umzusetzen - mehr als einmal zum Verdruss der CSU-Abgeordneten. Die Schul- und Bildungspolitik ist dafür ein beredtes Beispiel. Nicht von ungefähr verficht Seehofer den Ausbau der Instrumente direkter Demokratie im Bund wie in Europa und passt sich und seine Regierungsmannschaft an die in bayerischen Volksbegehren wie Abstimmungen sichtbar werdenden Politikpräferenzen der Bürger unter Aufgabe bisheriger Grundsatzpositionen an. Die Abschaffung der Studiengebühren ist dafür ebenso Beleg wie die aktuellen Diskussionen um die Einführung der Wahlfreiheit zwischen einem acht- oder neunjährigem Gymnasium, während die Regierungserklärung neue Schulreformen für die nächsten Jahre noch kategorisch ausgeschlossen hatte. $^{53}$

52 Vgl. Bayerische Staatsregierung, Regierungserklärung „Bayern. Die Zukunft“ des Bayerischen Ministerpräsidenten Horst Seehofer am 12. November 2013, http://www.bayern.de/Regierungserklaerungen-.1290.10489479/index.htm (Abruf am 14. Mai 2014).

53 Vgl. Frank Müller / Mike Szymanski, Spaenle in Erklärungsnot, in: SZ vom 29. Januar 2014, S. 30; Roland Preuß/ Johann Osel, G9 als Segen und Fluch, in: Süddeutsche.de vom 5. April 2014, http://www.sueddeutsche.de/bildung/geplante-gymnasialreform-g-als-segen-und-fluch-1. 1930041 (Abruf am 9. Mai 2014). 


\section{Zusammenfassung}

(1) Die Wahlbeteiligung stieg gegenüber der vorangegangenen Landtagswahl um 5,7 Punkte auf 63,6 Prozent; sie blieb damit aber um einiges hinter der Beteiligung bei der nachfolgenden Bundestagswahl (70,0 Prozent) zurück. Der aktuelle Anstieg, der sicherlich zu einem Gutteil der besonderen Mobilisierung während des fast zeitgleichen Verbundwahlkampfes für Land- und Bundestag geschuldet ist, sollte nicht über den generellen Abwärtstrend wie über die zwischen Ebenen und Gruppen bestehenden bezogen deutlichen Differenzen in den Raten konventioneller Partizipation hinwegtäuschen.

(2) Durch den Wahlsieg der CSU wurde die Abkehr von den Großparteien und die weitere Pluralisierung des Parteiensystems zeitweilig gestoppt. Allerdings erhielten Union und Sozialdemokraten zusammen gerade einmal zwei Drittel der abgegebenen Stimmen. Immerhin 14,0 Prozent der Stimmen entfielen auf Parteien, die an der Barriere der Sperrklausel scheiterten. Bei Wahlen ohne Sperrklausel wie bei der Kommunalwahl vom März 2014 setzte sich der Trend der Pluralisierung indes fort; ein ähnliches Ergebnis dürfte sich bei der Wahl zum Europäischen Parlament einstellen.

(3) Auf die drei oppositionellen Parlamentsparteien entfielen zusammen 38,2 Prozent der Stimmen, unverändert gegenüber der Wahl von 2008 und in den Gewichten nur geringfügig zugunsten der SPD verschoben. Aufgrund der Mandatsmehrheit der CSU sind die oppositionellen Einwirkungsmöglichkeiten eher geschwächt. Zudem sind Sozialdemokraten, Freie Wähler und Grüne in ihrer sozialen, kulturellen wie regionalen Verankerung in der Gesellschaft zu unterschiedlich, als dass sie in der Opposition programmatisch wie strategisch zusammenfinden könnten. So bleiben nur punktuelle Gemeinsamkeiten, mit denen man versuchen kann, die Alleinregierung der Union erfolgversprechend herauszufordern.

(4) Wahlsoziologisch bestätigte die Landtagswahl die Existenz der sozial wie kulturell begründeten Asymmetrie zugunsten der CSU. Diese hat Konsequenzen für die Wählerwanderungen, denn die Volatilität ist gerade in der bayerischen Wählerschaft keineswegs beliebig. Die Wechselwähler bleiben ihren sozial wie politisch-ideologisch definierten Lagern treu. Lagerübergreifender Wechsel ist unverändert gering.

(5) Die politische Agenda wird aufgrund der eindeutigen Mehrheitsverhältnisse im Landtag und der Geschlossenheit der Fraktion noch stärker als in der vorangegangenen Wahlperiode von der Staatsregierung und der CSU dominiert werden. Daher dürfte für die Opposition in den nächsten Jahren eher der direktdemokratische als der parlamentarische Weg Möglichkeiten eröffnen, alternative Policy-Positionen in praktische Politik umzusetzen. 